\title{
Einige unstetige stochastische Prozesse
}

\section{Doctoral Thesis}

Author(s):

Dalcher, Andreas

Publication date:

1956

Permanent link:

https://doi.org/10.3929/ethz-a-000089794

Rights / license:

In Copyright - Non-Commercial Use Permitted 
Prom. Nr. 2483

\title{
Einige unstetige stochastische Prozesse
}

\author{
VON DER \\ EIDGENÖSSISCHEN TECHNISCHEN HOCHSCHULE \\ IN ZÜRICH \\ ZUR ERLANGUNG
}

DER WURDE EINES DOKTORS DER MATHEMATIK

GENEHMIGTE

PROMOTIONSARBEIT

VORGELEGT VON

ANDREAS DALCHER

VON

PRATTELN (BL)

Referent: Herr Prof. Dr. W. Saxer

Korreferent: Herr Prof. Dr. A. Pfluger

BASE L

Buchdruckerei Birkhäuser AG.

1956 


\section{Einige unstetige stochastische Prozesse}

Von Andreas Dalcher, Zug

\section{Inhalt}

1. Kapitel: Allgemeines . . . . . . . . . . . . . . 3

$\S$ 1. Einleitung . . . . . . . . . . . . . . 3

$\S$ 2. Beispiele. . . . . . . . . . . . . . . . . 5

§ 3. Die Hauptformel . . . . . . . . . . . . . . . . . . . . 6

2. Kapitel: Lösung durch Integralgleichungen . . . . . . . . . . . . 7

§ 4. Herleitung der Integralgleichung . . . . . . . . . . . . 7

$\S 5$. Eigenschaften der Kerne und elementare Lösungsmethoden für Volterra-Gleichungen . . . . . . . . . . . . . . . . 9 9

§ 6. Anwendungen . . . . . . . . . . . . . . 15

3. Kapitel: Lösung durch die Laplace-Transformation . . . . . . . . 18

$\S$ 7. Herleitung der Differentialgleichung. . . . . . . . . . 18

§ 8. Lösung der Gleichungen erster Ordnung . . . . . . . . . . 19

$\S$ 9. Die Gleichungen höherer Ordnung im stationären Fall . . . . 22

§ 10. Partielle Gleichungen zweiter Ordnung . . . . . . . . . . 27

$\S 11$. Stationärer Zustand bei exponentiell verteilter Sprunghöhe. . 28

4. Kapitel: Diverses. . . . . . . . . . . . . . . . . . . 31

§ 12. Ansatz für den mehrdimensionalen Fall . . . . . . . . . . 31

§13. Berechnung von Näherungen . . . . . . . . . . . . 31

§ 14. Anmerkungen über Existenzsätze . . . . . . . . . . . . 33

Literaturverzeichnis . . . . . . . . . . . . . . 33

\section{Kapitel: Allgemeines}

\section{$\S 1$. Einleitung}

a) Das Problem. Gegeben sei eine gewöhnliche Differentialgleichung erster Ordnung. Die Zeit $t$ sei die unabhängige, $x$ die abhängige Variable. Die Gleichung kann in der Form

$$
\frac{d x}{d t}=\dot{x}(x, t)
$$


geschrieben werden. Wir betrachten nun den folgenden stochastischen Prozess $x(t)$.

In jedem Zeitintervall $(t, t+d t)$ besteht eine Wahrscheinlichkeit $w(x, t) d t$, dass $x$ einen Sprung ausführt. Wenn dieser zur Zeit $t$ stattfindet und wenn die abhängige Variable in $(t-0)$ den Wert $y$ angenommen hat, so hat $x$ unmittelbar nachher die Verteilung $G(y, x, t)$. Vom so erreichten Punkt $x$ aus folgt der Prozess bis zum nächsten Sprung der durch $(x, t)$ gehenden Lösung der Differentialgleichung. In Figur 1 sind einige Kurven der Lösungsschar und ein möglicher

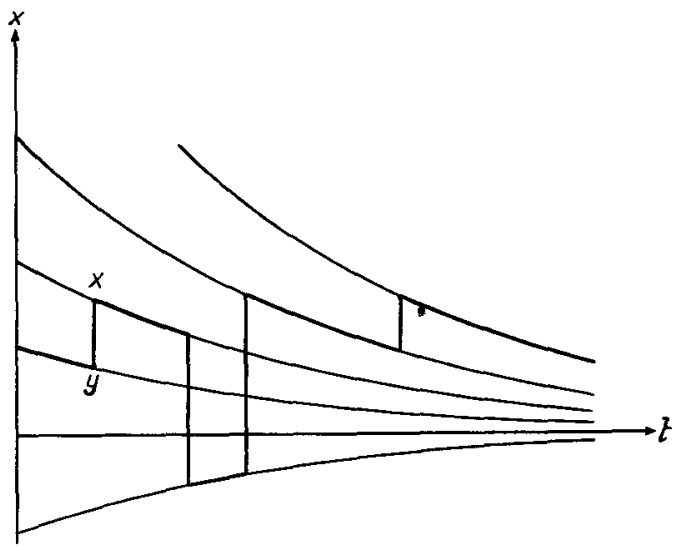

Figur 1

Möglicher Verlauf eines Prozesses.

Verlauf des Prozesses $x(t)$ dargestellt. Der Anfang des Prozesses sei durch die Werteilung $F(x, 0)$ gegeben.

Wir kennen also

die Differentialgleichung

die Dichte der Sprünge

$\dot{x}(x, t)$,

die Verteilung nach diesen

$w(y, t)$,

die Anfangsverteilung

$G(y, x, t)$,

und wollen berechnen ${ }^{1}$ )

$F(x, 0)$

die Verteilung von $x$ zu irgendeiner Zeit

$F(x, t)$.

b) Methoden und Resultate. Die Lösungen werden auf zwei Arten erhalten. Mit Integralgleichungen gewinnt man einen besondern Aspekt und allgemeinere Ergebnisse des "Queueing »-Problems und ähnlicher Fragen (vgl. KendaLL[6]²)). Durch die Laplace-Transformation lässt sich unter anderem das "Geräusch-

1) Auf das Problem der Verteilung im Funktionenraum [im Lehrbuch von Doos [3] mit $\Omega$ bezeichnet: $\left.x_{t}(\omega), \omega \in \Omega\right]$ treten wir hier nicht ein. Auch die Autokorrelation des Prozesses wird nicht untersucht.

2) Die Ziffern in eckigen Klammern verweisen auf das Literaturverzeichnis, Seite 33. 
problem» behandeln. Hier wird die Störung jedoch als unstetig angenommen, während in der Literatur das Hauptgewicht auf den kontinuierlichen Fall gelegt wird.

\section{§2. Beispiele}

a) Wir betrachten ein Reservoir in einer wasserarmen Gegend (zum Beispiel Jura). Dieses werde direkt durch Regenwasser und nicht durch eine Quelle gespiesen. $x(t)$ sei das Volumen des gespeicherten Wassers. Wir nehmen an, Regengüsse erfolgen mit einer Wahrscheinlichkeitsdichte $w(t)$ und seien nur von kurzer Dauer. Wenn das Wasser sofort in das Reservoir geleitet wird, so nimmt $x$ jeweils sprunghaft zu. $-\dot{x}(x, t)$ ist die pro Zeiteinheit gebrauchte Wassermenge. Bei leerem Reservoir ist $\dot{x}(0, t)=0$. Wenn mehr Wasser als die maximale Kapazität $A$ anfällt, so überläuft es sofort. Dies bedeutet $\dot{x}(x, t)=-\infty$ für $x \geqq A$. Im Intervall $0<x<A$ ist $\dot{x}$ negativ.

Ähnlich verhält es sich mit Stauseen für Kraftwerke. Die Bedingung, dass das Regenwasser sofort in den See gelangt, ist allerdings oft nicht erfüllt, besonders wenn Gletscher oder Wälder im Einzugsgebiet liegen. Ein stetiger Zufluss kann jedoch berücksichtigt werden, indem dieser zu $\dot{x}$ addiert wird. Für die folgenden Rechnungen muss aber $\dot{x}$ eine bestimmte Funktion von $x$ und $t$ sein. Weder der allfällige stetige Teil des Zuflusses noch der für die Krafterzeugung benötigte Abfluss dürfen ein stochastisches Element enthalten.

b) Im Lagerungsproblem ist $x(t)$ die Menge der zur Zeit $t$ gelagerten Güter. $w$ ist die Wahrscheinlichkeitsdichte, dass eine neue Sendung eintrifft. $G(y, x, t)$ ist die Verteilung von $x$ nach deren Ankunft, wenn vorher die Warenmenge $y$ betragen hat. Wir nehmen an, der Verkauf erfolge stetig. $-\dot{x}$ ist die Verkaufsgeschwindigkeit. Auch hier hat man die Bedingung

$$
\dot{x}(0, t)=0, \quad \dot{x}(x, t)<0 \quad \text { für } \quad x>0 .
$$

c) Auch das Warteproblem (queueing problem) kann in dieser Form dargestellt werden. $x(t)$ ist jedoch nicht die Anzahl wartender Personen, sondern die durch diese für den Bedienenden aufgespeicherte Arbeit. $w(y)$ ist die Wahrscheinlichkeitsdichte, dass sich jemand anschliesst, wenn die Länge der Schlange, ausgedrückt in Arbeit, $y$ beträgt. $G(x-y)$ ist die Verteilung der Arbeit, die ein Ankommender bringt. $-\dot{x}(x, t)$ ist die Geschwindigkeit, mit welcher der Bedienende arbeitet.

d) Ein Geiger-Müller-Zählrohr löst bei jedem registrierten Partikel einen kurzen Stromstoss aus. Dieser gelange auf einen $C R$-Kreis. Wie lautet die Wahrscheinlichkeitsverteilung der Spannung $x$ am Kondensator? Bei jedem Stromstoss nimmt letztere sprunghaft zu und folgt zwischen den Unstetigkeiten der Differentialgleichung

$$
\dot{x}=-\frac{x}{C R} .
$$

